\title{
EL TíTUlO TÉCNICO EN EL EMPLEO PÚBLICO
}

En la convicción de que un buen sistema educacional contiene diferentes niveles de formación, que configuran una red debidamente articulada y flexible, y que pone a disposición de los individuos diversas rutas de desarrollo a lo largo de la vida, en las últimas décadas las políticas públicas nacionales en esta materia se han planteado, entre otros desafíos, la promoción de la educación técnica. Los esfuerzos del gobierno por fomentarla se han concentrado principalmente en mejorar su disponibilidad de financiamiento ${ }^{1}$, en la búsqueda de convergencia entre la oferta académica de nivel técnico y los requerimientos provenientes del sector productivo ${ }^{2}$, y en la creación de sistemas de información sobre estas carreras y la inserción laboral de sus egresados ${ }^{3}$.

Tales esfuerzos se han visto reflejados en los cambios en términos de matrícula y variedad de programas ofrecidos. La matrícula en carreras técnicas, en el sistema de educación superior, ha aumentado en un 20\% desde 2004 a la fecha, observándose un alza de un 28\% en el número de inscritos en centros de formación técnica (CFT) durante el mismo periodo, lo que indica que el aumento de estudiantes ha sido proporcionalmente mayor en este tipo de instituciones. Asimismo, la gama de programas técnicos ha crecido ampliamente, al menos si se considera que en prácticamente todas las áreas del conocimiento existe la posibilidad de seguir este tipo de carreras $^{4}$.

1 El sistema de becas y créditos existente en Chile tradicionalmente ha privilegiado la formación universitaria en desmedro de las carreras técnicas. El acceso al crédito para los estudiantes de CFT e Institutos Profesionales (IP), la creación de la beca Nuevo Milenio, especialmente destinada para quienes inician estudios conducentes a títulos técnicos de nivel superior, así como el uso de la franquicia tributaria SENCE por las empresas cuyos empleados cursen estudios técnicos superiores, son algunas de las iniciativas gubernamentales que han mejorado la disponibilidad de recursos para el financiamiento de estudios de este tipo.

2 "Chilecalifica" es un programa gubernamental cuyo propósito es contribuir al desarrollo productivo del país y al mejoramiento de las oportunidades de progreso de las personas mediante la creación de un sistema de educación y capacitación permanente.

3 FuturoLaboral.

4 INDICES 2007. 
Es natural pensar, entonces, que las posibilidades ocupacionales de los egresados de la educación técnica sean amplias y variadas y que el Estado, en tanto empleador de recursos humanos, sea un destino potencial para muchos de quienes cuentan con un diploma técnico de nivel superior.

En este escenario de crecimiento, resulta interesante explorar en mayor detalle el contexto normativo en que se produce la contratación de técnicos por parte del Estado y reflexionar acerca de si sus condiciones de contratación son consistentes con los intentos por hacer atractivas las alternativas de estudios no profesionales. Cabe preguntarse si el Estado, como empleador, presenta condiciones favorables para la incorporación de técnicos a sus diferentes servicios.

Desde la perspectiva de las instituciones que otorgan títulos técnicos, parece útil conocer las exigencias normativas que deben cumplir sus egresados para desempeñarse en la Administración Pública, pues les permite entregar información oportuna a sus estudiantes (y postulantes) acerca del campo ocupacional al que podrían acceder, contribuyendo de esta manera a la transparencia del sistema. Les permite, también, analizar la conveniencia de recoger tales exigencias al momento de diseñar el currículo de determinados programas, en la medida que lo estimen pertinente.

Este artículo centra su análisis en el marco normativo que regula el título técnico en el sector público, sobre la base de un catastro de normas efectuado por el Consejo Superior de Educación (CSE) en relación con los requisitos formativos exigidos para el ingreso a la Administración Pública. A partir de dicho análisis, se pretende identificar los obstáculos que deben enfrentar los técnicos de nivel superior en el acceso a los cargos públicos y en el desempeño de su función, con miras a esbozar posibles mejoras que recojan, desde la perspectiva de contratación de personal en el sector público, las políticas que se vienen implementando para impulsar la educación superior. 


\section{Consideraciones metodológicas}

Entre 2005 y 2006, la Secretaría Técnica del CSE estudió los requisitos que fija el Estado para la contratación de personal en 151 órganos y servicios públicos. Se hizo una recopilación de las leyes y normas que regulan las plantas de personal de dichos servicios, se revisó el marco normativo que define las principales certificaciones académicas exigidas para el ingreso a la función pública, así como los criterios aplicados por la Contraloría General de la República en su interpretación ${ }^{5}$, y se elaboró un catastro con los datos obtenidos.

Dicho catastro reúne información de la normativa del personal de planta de los ministerios y servicios públicos creados para el cumplimiento de la función administrativa, regidos por el Título II de la Ley de Bases de Administración del Estado, y cuya estructura de personal es similar o equiparable a la organización básica definida en el Estatuto Administrativo, esto es, personal distribuido en plantas directivas, profesionales, técnicas, auxiliares y administrativas ${ }^{6}$.

Además, se han incluido dos tipos de plantas especiales que, si bien no responden a la estructura básica definida por el Estatuto Administrativo, se insertan en servicios públicos que sí se responden a ese ordenamiento ${ }^{7}$, pues resulta importante su análisis por cuanto tales plantas combinan simultáneamente requisitos académicos propios de las plantas de técnicos y de profesionales.

5 La Contraloría General de la República es el organismo encargado de fiscalizar la contratación de personal en el sector público y de interpretar las normas que disponen el pago de asignaciones y otros emolumentos en favor de los funcionarios.

6 Se han excluido las Fuerzas Armadas, Fuerzas de Orden y Seguridad Pública; empresas del Estado y empresas con participación estatal mayoritaria; poderes o funciones del Estado distintos del Ejecutivo: Poder Judicial y Poder Legislativo; municipalidades y órganos del Estado autónomos. Se han excluido, además, las siguientes plantas de personal: académicos de instituciones de educación superior estatales; personal del servicio exterior del Ministerio de Relaciones Exteriores, y del propio Ministerio y de sus servicios relacionados cuando cumple funciones en el extranjero, y personal de la planta de oficiales y vigilantes penitenciarios de Gendarmería de Chile.

7 Plantas de profesionales del área de la salud afectas a la Ley $N^{\circ} 15.076$ y plantas fiscalizadoras de la Fiscalía Nacional Económica, del Servicio Nacional de Aduanas, del Servicio de Impuestos Internos, de las superintendencias de AFP, de Bancos e Instituciones Financieras, de Valores y Seguros, de Seguridad Social y de la Dirección del Trabajo. 
De un total de 151 organismos públicos catastrados, se constató que 124 contaban con plantas de técnicos (82\%), 115 de las cuales contemplaban la exigencia de contar con títulos técnicos de nivel superior o medio para esos cargos $(93 \%)^{8}$. Asimismo, se observó que 60 organismos fijaban esta exigencia para sus plantas de directivos (40\%) y 44 la establecían para sus plantas de profesionales (29\%).

\section{1. ¿Qué se entiende por título técnico de nivel superior?}

De acuerdo con la Ley Orgánica Constitucional de Enseñanza (LOCE) ${ }^{9}$, el título técnico de nivel superior se otorga a un egresado de un CFT o de un IP que ha aprobado un programa de estudios de una duración mínima de 1.600 clases, y que le confiere la capacidad y los conocimientos necesarios para desempeñarse en una especialidad de apoyo al nivel profesional.

Desde el punto de vista sustantivo, esta certificación académica destaca por la formación eminentemente práctica de sus titulares, quienes se vinculan de manera inmediata con una actividad laboral determinada. De modo que, en la medida en que estos diplomas habilitan para realizar ciertas actuaciones o procedimientos específicos, son de apoyo al nivel profesional y no autónomos como los títulos profesionales ${ }^{10}$.

La definición legal indica que pueden otorgar títulos técnicos de nivel superior las universidades, los IP y los CFT. No obstante, la LOCE sólo menciona a los dos últimos como instituciones aptas para otorgar títulos técnicos de nivel superior. La Contraloría General de la República ha reconocido reiteradamente la potestad de las universidades en orden a impartir carreras técnicas y conferir los

8 Las disposiciones orgánicas de 7 organismos públicos no formulaban requisito especial alguno para el ingreso a sus plantas de técnicos.

9 Artículo 35 de la Ley Orgánica Constitucional de Enseñanza, LOCE.

10 Contraloría General de la República. Dictámenes No 31.806, de 2005; 380 de 2006; 98 de 2007; entre otros. 
correspondientes diplomas, considerando la ausencia de limitaciones expresas en este sentido dentro del conjunto de normas que fija la LOCE sobre la materia ${ }^{11}$.

Adicionalmente, el título técnico es el único dentro de nuestro sistema de títulos y grados que tiene una exigencia legal de duración mínima del programa de estudios que le da origen y que, por lo mismo, cuenta con un parámetro de duración que permite determinar qué certificaciones cumplen con las condiciones necesarias para calificarlas como títulos técnicos de nivel superior. La LOCE usa el criterio del número mínimo de clases (1.600) del programa de estudios conducente al diploma como un elemento determinante de la calidad de título técnico de nivel superior.

Sin embargo, no precisa qué se entiende por "clases", ni especifica si el término corresponde a horas de clases pedagógicas de 45 minutos o a horas cronológicas. Tampoco aclara si en ellas deben o no considerarse las horas destinadas a estudio individual, ayudantías, prácticas, seminarios o actividades de titulación. Si bien la Contraloría General de la República ha contribuido a esclarecer este punto, señalando que tales actividades no deben ser consideradas para los efectos del cómputo de las horas clases ${ }^{12}$, este criterio pareciera estar en continua revisión pues, en 2006, la misma Contraloría indicó que tales actividades sí debían considerarse en el cómputo final, en la medida en que el propio plan de estudios las defina como horas clases $^{13}$.

11 Contraloría General de la República. Dictámenes No 14.954 de 1994; 31.748 de 1995; 18.633 de 1996; 13.822 y 32.199 de 1998.

12 Contraloría General de la República. Dictámenes Nº 16.779 de 1998 y 21.337 de 1999.

13 Contraloría General de la República. Dictamen No 11.551 de 2006, a propósito del requerimiento de horas de clases (3.200) fijado por el DL $N^{\circ} 479$, de 1974, para el pago de la asignación profesional, señala que "el tiempo empleado en prácticas de estudios o prácticas profesionales, trabajos de título, giras de estudios, entre otras actividades, puede ser computado para los efectos de dar cumplimiento a la exigencia de horas de clases que contempla el mencionado decreto ley para el pago de la asignación profesional, siempre que en el correspondiente plan o programa de estudios de la respectiva carrera profesional se les haya dado a dichas actividades académicas el carácter de horas de clases fuera de aula". 
Esta referencia al número de clases no sólo es importante porque permita establecer si el título cumple o no los requisitos para ser considerado técnico de nivel superior, sino que, además, opera como un piso mínimo que deben cumplir los títulos profesionales para ser considerados como tales. De este modo, los programas de estudios conducentes a títulos profesionales no podrían tener una duración inferior que la exigida para los diplomas técnicos.

La revisión del concepto legal de "título técnico" permite visualizar los primeros obstáculos con que se podrían encontrar los poseedores de certificaciones técnicas al momento de optar a un cargo público.

En lo que respecta a las instituciones que pueden otorgar títulos técnicos, la interpretación sentada por la Contraloría, que entrega también a las universidades esta prerrogativa, podría impactar negativamente en la competitividad de quienes cuentan con títulos técnicos procedentes de CFT e IP, dado que es esperable que los empleadores (y los estudiantes) perciban que los técnicos universitarios poseen una formación más completa que los provenientes del resto de las instituciones.

Otra dificultad para el ingreso de técnicos de nivel superior a los cargos de la Administración del Estado podría derivarse de la falta de precisión en la definición legal del término "clases", ya que la estimación del cumplimiento de este requisito por parte del postulante queda supeditada a la interpretación que aplique el organismo público en particular y, en última instancia, al pronunciamiento de la Contraloría General de la República, que es la encargada de supervisar la observancia de tales exigencias.

Para complementar el examen de la definición de título técnico de nivel superior es necesario tener presente que también existe el título técnico nivel medio, como certificación académica que se confiere a los alumnos de los establecimientos de enseñanza media técnico-profesional al término de su educación secundaria. 
Los títulos técnicos de nivel medio tienen las siguientes características que los distinguen de los de nivel superior: (1) son otorgados por el Ministerio de Educación; (2) su certificación es equivalente a la licencia de enseñanza media científico-humanista; (3) dan cuenta de la obtención de las competencias propias de una especialidad, articuladas con el aprendizaje de la formación general de la enseñanza media, y (4) constituyen un ámbito de preparación inicial para la vida del trabajo y, a la vez, proporcionan las habilidades para continuar realizando estudios sistemáticos, sea que éstos se efectúen en el ámbito de la capacitación laboral o en el contexto de la educación superior ${ }^{14}$.

\section{El título técnico como requisito de ingreso a la Administración Pública}

A partir de la revisión de la normativa que regula el acceso a los cargos de la Administración Pública, plasmada en el catastro que da origen a este artículo, se puede constatar que el título técnico de nivel superior es el segundo requerimiento de mayor exigencia académica dentro del sistema, después del título profesional ${ }^{15}$.

Desde el punto de vista de la estructura de las plantas administrativas, este diploma se exige mayoritariamente para el ingreso a cargos pertenecientes a las plantas de técnicos, aun cuando también se presenta con bastante frecuencia como un requisito de acceso a cargos directivos y profesionales. Respecto de estos últimos, como se verá más adelante, los títulos técnicos de nivel superior otorgados por universidades pueden llegar a sustituir la exigencia de contar con un título profesional, permitiendo a quién posea dicha certificación desempeñarse en funciones profesionales.

14 Decreto No 220, de 1998, Ministerio de Educación. Capítulo VI: Objetivos Fundamentales Terminales para la Formación Diferenciada Técnico-Profesional en la Educación Media.

15 Ello sin considerar un número menor de cargos dentro de la Administración Pública que, con distintas variantes, exigen los grados académicos de licenciado, magíster o doctor. 
Las normas que regulan esta certificación académica para ingresar a un cargo público exigen usualmente estar en posesión de un título técnico genérico, sin exigir mayor especificidad. Cuando excepcionalmente se requiere formación en un área determinada, se trata de áreas como la economía, finanzas, administración, contabilidad, administración de recursos humanos, financieros o físicos, computación o informática, estadística, acciones de salud, entre otros.

En cuanto a la duración que deben tener los planes y programas de estudio conducentes a un título técnico, excepcionalmente se pide que éstos fluctúen entre los cuatro y diez semestres, solicitándose con mayor frecuencia programas de estudios de una duración inferior a seis semestres. Asimismo, se suele exigir determinada experiencia complementaria al título técnico de nivel superior, particularmente para acceder a cargos de las plantas de técnicos. La duración de la experiencia requerida puede variar entre seis meses y 15 años, solicitándose, como regla general, una experiencia de dos o tres años.

Excepcionalmente, la exigencia de experiencia tiene por objeto no sólo complementar el título técnico de nivel superior, sino suplirlo para ciertos cargos, en su mayoría pertenecientes a plantas de técnicos. En estos casos, la experiencia requerida oscila entre dos y ocho años.

En relación con la institución que debe otorgar el título técnico, frecuentemente se precisa que deben ser establecimientos de educación superior, esto es, universidades, IP o CFT, del Estado o reconocidos por éste. La regulación es aún más específica en algunos casos, solicitando expresamente que hayan sido otorgados "por centros de formación técnica"16, "por centros de formación técnica o institutos profesionales"17, o bien requiriendo puntualmente contar con un "título técnico universitario"18.

16 Planta de técnicos de la Comisión Nacional del Medio Ambiente, de la Dirección Nacional de Bibliotecas, Archivos y Museos, por ejemplo.

17 Planta de técnicos de la Subsecretaría de Bienes Nacionales, por ejemplo.

18 Plantas de profesionales de la Subsecretaría, de la Dirección General, de la Dirección de Planeamiento, de la Dirección de Contabilidad y Finanzas, de la Dirección de Vialidad, de la Dirección General de Aguas, de la Dirección de Obras Portuarias y de la Dirección de Obras Hidráulicas del Ministerio de Obras Públicas. Plantas profesionales de la Dirección General de Aeronáutica Civil y de los servicios de salud, por ejemplo. 
Cuando la normativa exige específicamente títulos técnicos de nivel superior otorgados por CFT, se ha interpretado que resultan igualmente habilitantes los entregados por IP y universidades. Lo mismo sucede cuando se exigen títulos técnicos otorgados por IP, entendiéndose que son también habilitantes los universitarios ${ }^{19}$.

Estas exigencias relativas a la institución de educación superior de la cual debe proceder el título, implican la exclusión de los títulos técnicos de nivel medio como credenciales idóneas para postular a dichos $\operatorname{cargos}^{20}$.

Sin embargo, para la mayor parte de las plantas de técnicos y de directivos se admiten indistintamente tanto los títulos técnicos de nivel superior como los de nivel medio, no obstante que los primeros supongan un mayor nivel de formación técnica que los segundos y que nuestro sistema educativo distinga claramente entre ambos tipos de certificaciones.

La admisibilidad simultánea de técnicos de nivel superior y de nivel medio para llenar un mismo cargo opera como consecuencia de que la normativa prescriba que se requiere contar, alternativamente, con un título técnico otorgado por un establecimiento de educación superior del Estado (técnico de nivel superior) o con un título técnico otorgado por un establecimiento de educación media técnicoprofesional del Estado o reconocido por éste (técnico de nivel medio). También tiene lugar cuando se exigen expresamente títulos técnicos de nivel medio, interpretándose que resultan igualmente habilitantes los de nivel superior, por cuanto éstos suponen una mayor exigencia formativa que los primeros ${ }^{21}$.

19 Contraloría General de la República. Dictamen No 55.596 de 2006

20 Esta exclusión del título técnico de nivel medio se observa en 80 de las 426 plantas que se incluyeron en el catastro (19\%). Así, se pudo observar que 16 plantas de técnicos, esto es, el 14\% del total de plantas técnicas que requieren el título técnico de nivel superior, contemplan cargos para los cuales se exige este diploma con exclusión del título técnico de nivel medio. Se observó, asimismo, que 22 plantas de directivos (37\%) y 42 plantas de profesionales (96\%) se encuentran en esta situación, destacándose particularmente en estas últimas la restricción a la alternancia de los títulos técnicos de nivel superior y medio.

21 Contraloría General de la República. Dictamen No 27.816 de 1986. 
En algunos servicios públicos, la normativa ha sido aún menos restrictiva en la exigencia de la formación técnica, permitiendo igualmente el ingreso a cargos de las plantas de técnicos a personas que cuenten con títulos técnicos de nivel superior como a quienes posean solamente la licencia de enseñanza media y hayan aprobado ciertos cursos, rendido determinados exámenes o cuenten con determinada experiencia.

Es el caso de los cargos técnicos de menor grado en los servicios de salud pública, a los cuales pueden ingresar personas que, contando con la licencia de enseñanza media, tienen aprobado un curso de formación de Auxiliar Paramédico de 1.500 horas, de acuerdo con los programas reconocidos por el Ministerio de Salud. Para estos cargos se admite, asimismo, la incorporación de personas que han aprobado un examen teórico y uno práctico, rendido en el propio Servicio de Salud, similar al que se someten quienes han cursado el referido curso de Auxiliar Paramédico, y de quienes, aun sin rendir tales exámenes, acreditan tener experiencia laboral en funciones afines, en el sector público o privado.

Similar situación ocurre en cargos que integran las plantas de técnicos de los organismos dependientes del Ministerio de Obras Públicas, para la mayor parte de los cuales se requiere solamente la licencia de enseñanza media y la aprobación de determinados cursos (programador, dibujante técnico, secretariado, operador de computador, geotecnia, digitador, observador meteorológico, entre otros), con o sin experiencia, dependiendo del cargo.

Estos ejemplos si bien parecen estar referidos a cargos puntuales dentro de la Administración Pública, se presentan en los 30 servicios de salud pública incluidos en el estudio y en 10 de los 13 organismos que conforman el Ministerio de Obras Públicas, los que representan el 32\% del total de las plantas de técnicos que se analizaron.

Como se puede apreciar, la formulación del requisito admite que el título técnico de nivel superior sea reemplazado por el 
título técnico de nivel medio e, incluso, por determinados años de experiencia, lo que podría dar lugar a que los servicios privilegien la incorporación de personas que, sin contar con estudios formales técnicos de nivel superior, han adquirido cierta experiencia dentro del propio organismo, desplazando a quienes cuentan con tal preparación.

\section{El título técnico y el título profesional}

Así como la mayor parte de los cargos públicos de las plantas de técnicos exigen contar alternativamente con un título técnico de nivel superior o con un título técnico de nivel medio, las plantas de profesionales, en forma excepcional, admiten indistintamente tantos títulos profesionales como títulos técnicos pero de nivel universitario.

En efecto, en 41 plantas profesionales se observó la existencia de cargos de menor grado respecto de los cuales la normativa evalúa como equivalentes los títulos profesionales y los títulos técnicos de nivel superior, siempre que éstos sean otorgados por universidades.

Ello sucede en las plantas de profesionales de los servicios de salud pública y otros organismos dependientes del Ministerio de Salud $^{22}$, organismos dependientes del Ministerio de Obras Públicas, la Comisión Nacional de Riego ${ }^{23}$, la Universidad de Santiago y la Corporación de Fomento de la Producción.

Con todo, se ha reconocido como extraordinaria la posibilidad de ingresar a plantas profesionales en virtud de títulos técnicos, así sean de carácter universitario, estableciéndose que, en todo caso, la procedencia de tal ingreso no confiere derecho a los beneficios remuneratorios asociados a un título profesional, y específicamente a la asignación profesional ${ }^{24}$. Es decir, una persona con título técnico

22 Central de Abastecimiento, Instituto de Salud Pública de Chile y Fondo Nacional de Salud.

23 El DFL N³-18834 de 1990, del Ministerio de Economía, Fomento y Reconstrucción, exige "titulo profesional de técnico universitario".

24 Contraloría General de la República. Dictamen No 39.020 de 2002. 
podrá ingresar a un cargo de planta profesional si así lo permite la ley, pero no obtendrá los beneficios propios del título profesional.

A su vez, se observó que en 35 de las 60 plantas de directivos que exigen títulos técnicos, resultan también habilitantes los títulos profesionales, los técnicos de nivel superior y los técnicos de nivel medio.

Se observa que la normativa tampoco favorece el ingreso de técnicos de nivel superior a las plantas de profesionales y directivos. Ello, porque en dichas plantas el ingreso de técnicos de nivel superior sólo tiene lugar tratándose de organismos y cargos puntuales, respecto de los cuales deben competir con quienes ostentan diplomas profesionales y siempre y cuando, como regla general, el título técnico sea universitario. Aun ingresando a tales plantas, los técnicos de nivel superior no gozan de los beneficios remuneratorios propios de los profesionales.

En las plantas de directivos, en tanto, usualmente el técnico de nivel superior no sólo entra en competencia con profesionales sino también con técnicos de nivel medio, de modo que esta planta de personal tampoco privilegia especialmente su ingreso a los cargos públicos.

\section{Diferencias de remuneración que afectan a los técnicos: la falta de una asignación especial por formación técnica}

Los cargos que conforman las plantas de técnicos usualmente tienen grados intermedios entre aquellos que pertenecen a las plantas de profesionales y los que conforman las de administrativos y auxiliares, de modo que sus remuneraciones suelen ser menores que las de los primeros y mayores que las de los segundos.

En la estructura de remuneraciones del personal técnico, a diferencia de lo que sucede con los profesionales, no se contempla 
una asignación especial para quienes cuentan con una certificación. Ello plantea una diferencia en la composición de las remuneraciones de profesionales y técnicos.

Los profesionales cuentan con un beneficio denominado "asignación profesional", que fluctúa entre el 30\% y el 70\% de su sueldo base, dependiendo del grado, lo que constituye alrededor de un $20 \%$ del sueldo bruto total, mientras que los técnicos no cuentan actualmente con un beneficio de similar naturaleza.

En $1974^{25}$ se creó, para la Administración Pública chilena, la asignación profesional, beneficio económico que se otorga a los funcionarios públicos que, cumpliendo con ciertos requisitos que establece la ley, cuentan con un título de carácter profesional.

Originalmente, este beneficio se otorgó además a los funcionarios que, sin tener un título profesional, ocupaban cargos cuyo ejercicio requería estar en posición de tal diploma o para cuyo desempeño se exigía, alternativamente, estar en posesión de título profesional universitario u otro requisito de especialidad o conocimiento. Sin embargo, en $1977^{26}$ se derogaron las disposiciones que otorgaban la asignación profesional a estos funcionarios, quedando reservada sólo a quienes poseían título profesional.

Entre 1993 y 1999, la Contraloría General de la República, al momento de evaluar el cumplimiento de los requisitos para determinar la procedencia de la asignación profesional, estimó que determinados títulos técnicos de nivel superior conferidos por universidades tenían la calidad de títulos profesionales universitarios $y$, en consecuencia, habilitaban a sus poseedores para percibir la asignación correspondiente. El organismo contralor fundamentó tal criterio en que, de acuerdo con lo informado por las propias universidades que habían otorgado estos diplomas, ellos provenían de programas de estudios cuyo nivel y contenidos conferían una

25 DL 479, de 1974, artículo $3^{\circ}$.

26 DL 2056, de 1977, artículo $3^{\circ}$. 
formación general y científica necesaria para un adecuado desempeño profesional, entendiendo con ello que, más allá de la denominación del título dada por la institución, el nivel de formación era equivalente al profesional ${ }^{27}$.

A partir de $1999^{28}$, y sobre la base de lo informado por el Ministerio de Educación, la Contraloría modificó su criterio, estableciendo que los títulos técnicos de nivel superior no pueden, por su propia naturaleza, ser considerados como profesionales y, por tanto, habilitar a quienes los posean para percibir la asignación profesional u otros beneficios administrativos que requieran estar en posesión de un diploma profesional, no obstante haber sido otorgados por universidades.

Posteriormente, la Ley $N^{0}$ 19.699, de 2000, vino a esclarecer la procedencia de la asignación profesional, estableciendo que serán considerados títulos profesionales habilitantes los otorgados por una universidad o IP a quienes hubieren cursado programas de estudios de un mínimo de seis semestres académicos y 3.200 horas de clases.

La misma ley reguló la situación de los funcionarios que, contando con títulos técnicos de nivel superior y con base en la primera interpretación de la Contraloría, habían comenzado a percibir la asignación profesional, viéndose afectados por el cambio de criterio ${ }^{29}$. Al respecto, se estableció que tales funcionarios gozarían una asignación especial equivalente al monto de la última asignación profesional percibida.

En consecuencia, salvo lo indicado en el párrafo anterior, no existe en nuestro sistema una "asignación técnica" que recompense el mejoramiento de la formación de los funcionarios públicos a través de la obtención de títulos técnicos de nivel superior.

27 Contraloría General de la República. Dictámenes № 34.454 y 11.874 de 1993 y 14.307 de 1994.

28 Contraloría General de la República. Dictamen No 42.334 de 1999.

29 La Ley $N^{\circ}$ 19.882, en su artículo sexagésimo octavo, interpretó las disposiciones de la Ley $\mathrm{N}^{\circ} 19.699$. 
Por otra parte, se ha estimado que el pago de la asignación profesional opera con independencia de la planta de personal a la que pertenezca el funcionario que cuenta con un título profesional ${ }^{30}$. La posibilidad de obtener este beneficio ha despertado el interés de los funcionarios por lograr una certificación, a lo que muchos servicios han respondido mediante convenios con instituciones de educación superior, particularmente universidades. Ello refleja un claro incentivo para que tanto el personal técnico como el auxiliar y administrativo obtenga títulos profesionales.

Todo lo anterior muestra de qué manera se privilegia la formación profesional por sobre la técnica, sin que se considere necesariamente el tipo de cargo que sirve el funcionario ni el beneficio real que puede generar para el desempeño de su función la obtención de un diploma de esa naturaleza.

\section{Plantas técnicas en organismos públicos de reciente data}

En diversos organismos públicos, la tendencia a priorizar la profesionalización de la función pública ha operado, en cierto modo, en desmedro del rol de las plantas de técnicos, reduciendo el número de funcionarios que sirven en estas plantas o, simplemente, no contemplándolas.

Este fenómeno se observa en casi todos los servicios y organismos públicos creados a partir de 2000. De un total de once organismos nuevos, cinco no tienen plantas de técnicos: Servicio Nacional del Adulto Mayor (2002), Unidad de Análisis Financiero (2003), Dirección Nacional del Servicio Civil (2003), Superintendencia de Casinos de Juegos (2005) y Superintendencia de Salud (2005).

La Subsecretaría de Redes Asistenciales (2005) y la Dirección de Compras y Contratación Pública (2003) contemplan plantas de

30 Contraloría General de la República. Dictamen №32.242 de 1999. 
técnicos con un solo funcionario. No obstante, estos organismos tienen respectivamente 53 y 18 funcionarios de planta en total, 34 y siete de los cuales pertenecen a la planta de profesionales.

Por su parte, la Agencia Nacional de Inteligencia (2004) y la Fiscalía Nacional Económica (2005) tienen plantas de técnicos con sólo dos funcionarios, aun cuando sus plantas cuentan con un total de 98 y 50 funcionarios respectivamente, de los cuales 34 y 17 corresponden a cargos profesionales.

El Instituto Nacional de Deportes de Chile (2001) y el Consejo Nacional de la Cultura y de las Artes (2003) son los organismos con plantas de técnicos más numerosas, contemplando 28 y nueve funcionarios, de un total de 351 y 98 , respectivamente.

Con anterioridad a 2000, también existían un conjunto de organismos que no contemplaban plantas de técnicos en su personal $^{31}$. En otros organismos, en tanto, se puede apreciar una tendencia a la reducción de las plantas de técnicos. Así por ejemplo, la Ley $N^{\circ}$ 18.890, de 1990, dispuso la extinción de la Planta de Técnicos de la Corporación de Fomento de la Producción, a medida que dichos cargos fueran quedando vacantes.

Esta decisión tiene importantes consecuencias, entre otras, el que no se permita la incorporación mediante contrata a personal con este tipo de formación, por cuanto se ha interpretado que el personal a contrata debe cumplir con los requisitos exigidos en la planta respectiva ${ }^{32}$. Ello genera un problema administrativo para los servicios públicos, dado que no tienen la posibilidad de contar con

31 Dirección General de Obras Públicas (1888), Subsecretaría del Trabajo (1959), Subsecretaría de Agricultura (1960), Dirección de Presupuestos (1960), Fiscalía del Ministerio de Obras Públicas (1964), Comisión Nacional de Riego (1975), Comisión Chilena del Cobre (1976), Dirección Nacional de Fronteras y Límites del Estado (1978), Servicio Nacional de Geología y Minería (1980), Dirección Administrativa del Ministerio de Defensa (1990), Consejo Superior de Educación (1990), Instituto Nacional de la Juventud (1991), Fondo Nacional de la Discapacidad (1994), y todas las superintendencias, salvo la de Quiebras.

32 Contraloría General de la República. Dictámenes № 8.940 de 2007, 32.781 de 1996, 14.878 de 2004 y 60.314 de 2006. 
personal calificado en el desempeño de un conjunto de funciones que requieren del conocimiento técnico, como, por ejemplo, el manejo de modernas tecnologías de la información. Asimismo, afecta el desarrollo de la carrera funcionaria, ya que, en los servicios que carecen de plantas técnicas, el personal administrativo de grado más alto sólo puede ascender accediendo a cargos de las plantas de profesionales, para los cuales se exige contar con diplomas cuya obtención plantea un costo mayor en tiempo y recursos que los títulos técnicos.

\section{Comentarios finales}

A partir de este análisis, se ha podido observar que, si bien se han invertido importantes esfuerzos y recursos en fomentar la formación de técnicos de nivel superior en nuestro país, el Estado ha omitido concebirse como una importante fuente de trabajo para los mismos técnicos que promueve formar.

La normativa que rige las plantas de personal y los requisitos formativos de ingreso a los cargos públicos da cuenta de un sistema de contratación de recursos humanos cerrado y rígido, regulado por un conjunto de prolíferas, generalmente antiguas y a veces confusas disposiciones. Este sistema se ha visto superado por la realidad de las necesidades de los propios servicios y por la variación de competencias que ha ido procurando la oferta académica.

Revisadas las normas de contratación de técnicos, y considerando, además, el marco regulatorio que fija la LOCE para esta certificación académica, se han detectado ciertas condiciones que ponen en una situación de relativa desventaja a quienes poseen un título técnico de nivel superior, en términos del acceso al cargo público, a las remuneraciones a las que pueden aspirar y a la probabilidad de desarrollar su experticia técnica en la administración del Estado.

En primer lugar, la obtención de un título técnico de nivel superior no parece reportar, usualmente, mejores condiciones de 
contratación para sus titulares, por cuanto deben competir en iguales términos con los técnicos de nivel medio para el ingreso a cargos de las plantas directivas y técnicas, y sólo en casos excepcionales pueden acceder a las plazas profesionales en virtud del diploma que poseen. Incluso, para determinados cargos técnicos, la normativa permite el ingreso de personas que cuentan solamente con licencia de enseñanza media científico-humanista y determinados cursos, o con cierta experiencia.

Lo anterior se traduce en que, a pesar de que los títulos técnicos de nivel superior y los títulos técnicos de nivel medio constituyen certificaciones académicas de diversa índole, desde el punto de vista del Estado empleador tendrían igual valor y supondrían similar idoneidad para habilitar el ingreso a determinados cargos.

Por su parte, en las plantas profesionales en que el título técnico es una alternativa al diploma profesional, la competitividad del técnico se ve restringida no sólo por el tipo de formación con la que se compara, sino porque, como regla general, sólo se aceptan aquellos técnicos provenientes de universidades.

En segundo término, la tendencia a no considerar cargos técnicos o reducir su número en la estructuración de las plantas de personal de los organismos públicos de reciente creación plantea un obstáculo para el ingreso de técnicos a plazas en las que podrían desempeñarse dentro del ámbito para el que fueron capacitados, así como el inconveniente para el propio servicio de contratar en forma permanente personal técnico calificado.

Además, y más allá del espectro de cargos a que pueden acceder los egresados de la formación técnica, se pudo constatar que la propia definición de título técnico presenta complejidades para determinar si procede o no el ingreso de un técnico a un cargo de la Administración del Estado que requiera específicamente ese nivel de formación. Ello por cuanto la LOCE, si bien proporciona una definición sustantiva del título técnico acompañada de un parámetro objetivo que sirve para 
determinar su existencia (1.600 clases), no define qué debe entenderse por "clases", ni especifica cuál es la duración de las "horas".

Será cada servicio público en particular y, en última instancia, la Contraloría General de la República los que precisen los alcances de la definición de título técnico para determinar si procede la incorporación de un postulante. No obstante, la decisión de la Contraloría es producto del análisis de la certificación de cada candidato, teniendo en cuenta, en aquellos casos en que se plantea la duda, la manera en que se encuentran formulados los planes y programas de estudio que conducen al título técnico. Por lo tanto, son las propias instituciones de educación superior las que aclaran cómo cada uno de sus programas de estudio cumple con la exigencia legal de 1.600 clases. De ahí que se observen diferentes interpretaciones aplicadas por la Contraloría en cada evaluación.

En consecuencia, la falta de una norma legal expresa que determine la definición y alcance del término "clases" presenta algún grado de dificultad para el ingreso de técnicos al sector público. Sería de utilidad contar con una interpretación más uniforme en esta materia, la que podría construirse a partir de la opinión de las propias instituciones educativas y los organismos del Estado involucrados. Ello evitaría la incertidumbre que se produce para el titular de la certificación y para el servicio que lo contrata.

Por último, respecto de los beneficios económicos a los que pueden aspirar los técnicos de nivel superior, se puede señalar que el establecimiento de la asignación profesional, como una retribución a la obtención de títulos profesionales, incentiva la profesionalización de los funcionarios públicos, con prescindencia de las plantas y tipo de cargos que éstos sirven. Ello porque la ley otorga esta asignación a todos quienes cuentan con títulos profesionales o los obtienen mientras desempeñan el cargo, cualquiera sea la planta a la que pertenezcan.

Si bien la profesionalización de la función pública reporta beneficios tanto educacionales como socioeconómicos para el 
personal, este fenómeno deviene en que la formación profesional a la que aspiran los funcionarios no se condiga necesariamente con el tipo de tareas que deben desempeñar en los cargos de las plantas administrativas, auxiliares e incluso técnicas a que pertenecen. Además, la obtención de un título profesional no asegura a su poseedor la posibilidad de obtener un ascenso en la carrera funcionaria en el mismo servicio, lo que trae como consecuencia la existencia de profesionales que permanecen más allá de lo esperado en las plantas auxiliares y administrativas.

Probablemente, la existencia de una asignación técnica de aplicación general permitiría cierta gradualidad en la formulación de exigencias formativas para la obtención de beneficios económicos, incentivando la tecnificación del personal administrativo y auxiliar, e incluso técnico que no cuente con este tipo de certificaciones. A su vez, una iniciativa de este tipo permitiría una administración más eficiente de los recursos humanos en el sector público, mejorando la gestión de los propios servicios, los que contarían con funcionarios calificados en aquellos cargos que requieren precisamente experticia técnica.

En conclusión, se puede apreciar que las normas de contratación de recursos humanos en el sector público no se han puesto en sintonía con los esfuerzos invertidos en la última década en la promoción de la formación de técnicos en Chile. Ello plantea la necesidad de revisar las disposiciones que estructuran las plantas de personal de los organismos públicos, que fijan requisitos para el ingreso a tales cargos y que establecen beneficios económicos, como una retribución a aquellos funcionarios que mejoran su formación optando por la educación superior técnica.

Recibido: 14 de agosto de 2007

Aceptado: 5 de noviembre de 2007 